\title{
Diagnostic sensitivity of SILVAMP TB-LAM (FujiLAM) point-of-care urine assay for extra-pulmonary tuberculosis in people living with HIV
}

To the Editor:

Diagnosing tuberculosis (TB) in people living with HIV (PLHIV) remains challenging in part, because of its diversity of clinical manifestations, including high rates of extra-pulmonary and disseminated disease [1]. In particular, disseminated TB, involving multiple organ systems, is associated with high mortality but often presents non-specifically, which may hinder prompt diagnosis [2, 3]. Xpert MTB/RIF (Xpert; Cepheid, Sunnyvale, CA, USA), is currently recommended by the World Health Organization (WHO) as the first line assay for evaluating a subset of extra-pulmonary TB disease (EPTB) manifestations [4]. To detect specific forms of EPTB, such as pleural TB, TB meningitis or TB lymphadenitis, Xpert may require an invasive sample to be collected, which often limits its use for EPTB detection to hospitals where appropriate equipment is available and invasive sampling can be safely performed. Furthermore, even when concomitant pulmonary disease is present, it can be very difficult to obtain sputum in the sickest HIV patients to submit for Xpert testing $[5,6]$. Therefore, an urgent priority for improving TB detection among PLHIV remains the development of rapid, point-of-care (POC) assays that use an easily obtainable clinical specimen, such as urine, and that have good diagnostic accuracy for both pulmonary and EPTB, including disseminated disease [7].

The commercially available Alere Determine TB LAM (AlereLAM; Abbott, Chicago, IL, USA) assay is a rapid, inexpensive, urinary POC TB test [8]. While its use is associated with a mortality benefit in severely ill and immunocompromised PLHIV [9, 10], it has only moderate sensitivity that is limited to patients with low CD4 counts, which has led to limited programmatic uptake [11]. We have previously reported on the Fujifilm SILVAMP TB LAM (FujiLAM; Fujifilm, Tokyo, Japan) POC assay that, similar to AlereLAM, detects the presence of lipoarabinomannan (LAM) in urine [12]. It offers on average 30\% improved sensitivity for detecting TB (independent of whether it is PTB or EPTB) compared to AlereLAM across subgroups stratified by CD4 strata, while maintaining high specificity. Here we report the sensitivity of FujiLAM in comparison to AlereLAM specifically for detecting EPTB in the same patient cohorts.

This post hoc analysis utilised data from two previously published, prospective cohort studies of adults (>18 years) living with HIV who were admitted to South African district hospitals on the outskirts of Cape Town $[13,14]$. Cohort A enrolled patients without a current TB diagnosis regardless of presenting signs or symptoms, and independent of CD4 count [13]. Cohort B enrolled patients with a CD4 count $<350$ cells. $\mu \mathrm{L}^{-1}$ in whom TB was considered the most likely diagnosis on admission [14]. A third previously published cohort was not included in the present analysis as it excluded patients with exclusively extra-pulmonary TB disease [12]. Informed consent was obtained from patients who had capacity or regained capacity and all study-related activities were approved by the Human Research Ethics Committee of the University of Cape Town.

Patients were systematically evaluated for the presence of TB. Whenever possible, patients provided two sputum samples, a blood sample and a urine sample for mycobacteriology; those unable to produce sputum or urine samples were not excluded from study enrolment. Sputum specimens were tested using

@ERSpublications

FujiLAM point-of-care rapid urine test for TB detects a large proportion of pulmonary and traditionally difficult-to-diagnose extra-pulmonary forms of TB in hospitalised patients with advanced HIV http://bit.ly/36Dl0Eo

Cite this article as: Kerkhoff $\mathrm{AD}$, Sossen $\mathrm{B}$, Schutz $\mathrm{C}$, et al. Diagnostic sensitivity of SILVAMP TB-LAM (FujiLAM) point-of-care urine assay for extra-pulmonary tuberculosis in people living with HIV. Eur Respir J 2020; 55: 1901259 [https://doi.org/10.1183/13993003.01259-2019]. 
smear fluorescence microscopy, mycobacteria growth indicator tube (MGIT) liquid culture (Becton Dickinson, Franklin Lakes, NJ, USA), and Xpert MTB/RIF Version G4. Blood specimens were tested using BACTEC Myco/F Lytic culture (Becton Dickinson, Franklin Lakes, NJ, USA). Sediments from urine specimens were tested using Xpert after centrifugation of 30-40 mL. The routine clinical team obtained additional specimens (sputum and non-sputum) as clinically indicated. FujiLAM and AlereLAM were performed on biobanked urine samples according to manufacturers' instructions and read by two investigators blinded to patient status and all other test results [12]. Microbiologically confirmed TB was defined by the detection of $M$. tuberculosis on any clinical specimen using either culture or Xpert. All patients with microbiologically-confirmed TB were classified into one of three mutually-exclusive groups: pulmonary TB (PTB) (TB detected in sputum only), EPTB (TB detected in extra-pulmonary specimen(s) only), or PTB+EPTB (TB detected in both sputum and at least one extra-pulmonary specimen). The sensitivity (and corresponding 95\% confidence intervals) of FujiLAM and AlereLAM was calculated for each form of TB as well as for individual forms of EPTB.

Of 1079 eligible patients, 111 had a TB status that could not be classified, 90 did not have urine samples and six had missing urine results; therefore, 872 patients (420 from cohort A and 659 from cohort B) had complete results and were included in this analysis. The median age was 36 (IQR 30-43) years, 54\% were female, the median CD4 count was 84 (IQR 32-188) cells $\mu \mathrm{L}^{-1}$, and $45 \%$ had previously been treated for TB. Among 872 patients, 553 (138 from cohort A and 415 from cohort B) had microbiologically confirmed TB (prevalence 56\%) on at least one specimen, 88 (37 from cohort A and 51 from cohort B) had possible TB and 231 (189 from cohort A and 42 from cohort B) had no evidence of TB. Of those with confirmed TB, 126 (23\%) out of 553 had PTB, 156 (28\%) out of 553 had EPTB, and 271 (49\%) out of 553 had both PTB+EPTB. The urine LAM assays performed best in those with PTB+EPTB, with FujiLAM detecting 91\% (95\% CI 8794; 246 out of 271) of cases compared with $61 \%$ (95\% CI 55-67; 165 out of 271) using AlereLAM (figure 1a). In patients with PTB or EPTB only, FujiLAM detected 60\% (95\% CI 51-69; 76 out of 126) and 67\% (95\% CI $59-75 ; 105$ out of 156) of cases, respectively, which was compared with 19\% (95\% CI 12-27; 24 out of 126) and $41 \%$ (95\% CI 33-49; 64 out of 156), respectively for AlereLAM (figure 1a).

The sensitivity for FujiLAM across different extra-pulmonary forms of TB disease ranged from 47 to $94 \%$ as shown in figure $1 \mathrm{~b}$. Notably, FujiLAM detected TB in $94 \%$ (95\% CI 90-97) of patients with TB mycobacteraemia and $88 \%$ (95\% CI 84-92) of those with TB confirmed by urine Xpert or culture. It also demonstrated moderate sensitivity in patients with microbiologically-confirmed pleural TB (68\%; $95 \%$ CI 55-80) and with TB meningitis (47\%; 95\% CI 24-71). AlereLAM's sensitivity ranged from 16 to $70 \%$ and performed best in those with TB mycobacteraemia (70\%; 95\% CI 64-76) and TB confirmed by urine Xpert or culture (61\%; 95\% CI 55-67).

Overall, FujiLAM showed substantially higher sensitivity over the commercially available AlereLAM, for detecting both pulmonary and extra-pulmonary TB in HIV inpatients. This suggests that FujiLAM may have clinical utility as a first-line test for the rapid detection of TB in HIV patients, independent of disease location. Given that a large proportion of patients with HIV-associated TB have EPTB and a diagnosis may only be possible using a non-sputum sample that may be challenging to obtain, an up-front FujiLAM test could substantially reduce the time to diagnosis. FujiLAM was able to detect TB in 67\% (105 out of 156) of patients who could not produce a sputum sample or did not have evidence of pulmonary disease; such patients comprised $28 \%$ of the study cohort.

FujiLAM performed best in those with TB mycobacteraemia as well as those with concomitantly positive sputum and non-respiratory cultures, detecting $>90 \%$ of cases. Mycobacterium tuberculosis bacteraemia is one of the most common blood stream infections among PLHIV in sub-Saharan Africa [3] and such patients have an extremely high mortality risk. FujiLAM's excellent performance in those with mycobacteraemia suggests a mechanistic association between disease dissemination and urinary LAM. This finding is supported by our recent study that showed a good association between detection of LAM in urine and serum of TB patients, independent of HIV status [15]. However, even for patients with forms of disease such as pleural TB and TB meningitis that may be compartmentalised, FujiLAM had moderate sensitivity, which could add substantial benefit in these cases. Taken together, these findings suggest that LAM antigenuria is likely indicative of glomerular filtration of circulating LAM (or LAM fragments) in addition to renal TB [16]. Further research, that aims to detect LAM with ultra-sensitive platforms, as well as characterisation of LAM structure in urine, is needed to better understand the mechanisms by which LAM enters the bloodstream and urine. This may help to further refine urine-based diagnostics and catalyse the development of blood-based assays. As the overall load of mycobacteria is expected to be higher in HIV-positive patients, our findings should not be generalised to HIV-negative individuals with EPTB.

Patients were not systematically evaluated for the presence of EPTB beyond mycobacterial blood cultures and urine Xpert; additional systematic sampling (e.g. pleural fluid or cerebrospinal fluid) could not be 


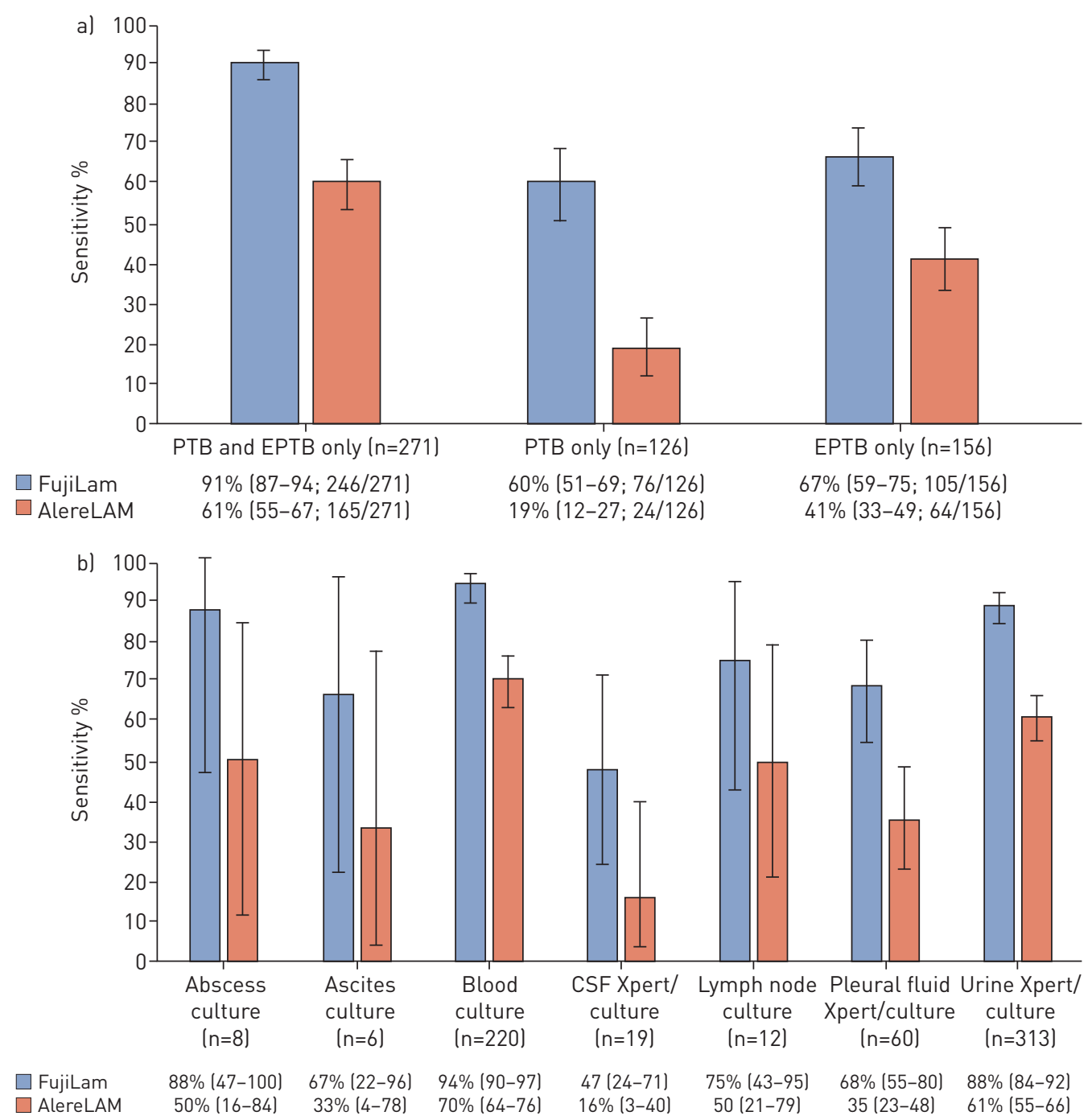

FIGURE 1 The diagnostic sensitivity of FujiLAM and AlereLAM by (a) type of tuberculosis disease (pulmonary, extra pulmonary or both; $n=553$ ), (b) site of disease involvement in patients with confirmed extra-pulmonary tuberculosis (EPTB). Bars represent 95\% confidence intervals. The numbers in parenthesis denote $95 \%$ confidence intervals. Of note, the same patient may have multiple sites of confirmed disease le.g., pulmonary, blood, urine, etcl. Sputum, blood and urine were obtained from all patients whenever possible and additional specimens were obtained at this discretion of routine medical team, however the ability to produce sputum was not a requirement for study entry. This analysis was limited to among those with both FujiLAM and AlereLAM results available. PTB: pulmonary tuberculosis.

justified as it would require invasive sampling where this was not clinically indicated. Coupled with challenges in universally obtaining clinically indicated samples (e.g. sputum), some misclassification of TB category (PTB, EPTB or PTB+EPTB) is likely present. Furthermore, because the overall cohort was severely immunocompromised (increasing the likelihood of disease dissemination) and because those with lower, site-specific mycobacterial burdens (CSF or pleural fluid) may not have been diagnosed by Xpert or culture, the true sensitivity of urinary FujiLAM (and AlereLAM) for localised extra-pulmonary disease is possibly an overestimate and may not be generalisable to all patients with these forms of disease. However, the additional specimens collected by the routine clinical team mirrored common practice in settings with a high-burden HIV-associated TB and followed clinical symptomatology. Finally, we did not evaluate the specificity of FujiLAM for specific disease forms given a lack of systematic EPTB sampling. We have previously reported the cohort-specific specificity as well as the estimated specificity using a Bayesian bivariate random-effects model in three cohorts: using a composite reference standard, the specificity of FujiLAM was 95.7\% (95\% CI 92.0-98.0\%) compared with 98.2\% (95\% CI 95.7-99.6\%) for AlereLAM [12].

In conclusion, our results suggest that the POC FujiLAM has good sensitivity for detecting both pulmonary and extra-pulmonary forms of TB in patients with advanced HIV, in which conventional 
diagnostics may be slow, require infrastructure and equipment or rely on samples that are difficult to obtain. While appropriate sampling should still be undertaken to allow for drug susceptibility testing, FujiLAM may be an appropriate first microbiological TB investigation for all hospitalised PLHIV, allowing for more rapid initiation of anti-TB therapy.

Andrew D. Kerkhoff $\circledast^{1,9}$, Bianca Sossen $\oplus^{2,3,9}$, Charlotte Schutz ${ }^{2,3}$, Elena Ivanova Reipold ${ }^{4}$, Andre Trollip ${ }^{4}$, Emmanuel Moreau, Samuel G. Schumacher $\oplus^{4}$, Rosie Burton, Amy Ward ${ }^{2,3}$, Mark P. Nicol ${ }^{6,7}$, Graeme Meintjes $^{2,3}$, Claudia M. Denkinger ${ }^{4,8,10}$ and Tobias Broger $\oplus^{4,10}$

${ }^{1}$ Division of HIV, Infectious Diseases and Global Medicine at Zuckerberg San Francisco General Hospital and Trauma Center, Dept of Medicine, University of California, San Francisco, CA, USA. ${ }^{2}$ Wellcome Centre for Infectious Diseases Research in Africa, Institute of Infectious Disease and Molecular Medicine, University of Cape Town, Cape Town, South Africa. ${ }^{3}$ Dept of Medicine, Faculty of Health Sciences, University of Cape Town, Cape Town, South Africa. ${ }^{4}$ FIND, Geneva, Switzerland. ${ }^{5}$ Southern African Medical Unit, Médecins sans Frontières, Cape Town, South Africa. ${ }^{6}$ Division of Infection and Immunity, School of Biomedical Sciences, University of Western Australia, Perth, Australia. ${ }^{7}$ Division of Medical Microbiology, University of Cape Town, Cape Town, South Africa. ${ }^{8}$ Division of Tropical Medicine, University of Heidelberg, Heidelberg, Germany. ${ }^{9}$ Contributed equally. ${ }^{10}$ Contributed equally.

Correspondence: Andrew D. Kerkhoff, University of California, San Francisco, Division of HIV, Infectious Diseases and Global Medicine, 1001 Potrero Avenue, San Francisco, California, 94110, USA. E-mail: andrew.kerkhoff@ucsf.edu

Received: 24 June 2019 | Accepted after revision: 18 Oct 2019

Acknowledgments: The investigators are grateful to the clinical and administrative staff of the Western Cape Department of Health as well as the patients who contributed to this data. The authors also wish to thank the late Stephen D. Lawn who designed and led the Cohortl study.

Author contributions: T. Broger, B. Sossen, A.D. Kerkhoff, C. Schutz, E.I. Reipold, M.P. Nicol, G. Meintjes and C.M. Denkinger designed the parent study and it was overseen by T. Broger, B. Sossen, A. Trollip, M.P. Nicol, G. Meintjes and C.M. Denkinger. B. Sossen, A.D. Kerkhoff, C. Schutz, R. Burton, A. Ward and G. Meintjes coordinated the individual study sites. A.D. Kerkhoff, T. Broger and C.M. Denkinger designed the statistical analysis and A.D. Kerkhoff undertook the statistical analyses. A.D. Kerkhoff, T. Broger and B. Sossen developed the first manuscript draft. All authors contributed to interpretation of data and editing of the article and approved the final version of the manuscript before submission.

Conflict of interest: A.D. Kerkhoff has nothing to disclose. B. Sossen has nothing to disclose. C. Schutz has nothing to disclose. E.I. Reipold has nothing to disclose. A. Trollip has nothing to disclose. E. Moreau reports grants from GHIT and KfW, during the conduct of the study. S.G. Schumacher is an employee of FIND. FIND is a non-for-profit foundation, whose mission is to find diagnostic solutions to overcome diseases of poverty in LMICs. It works closely with the private and public sectors and receives funding from some of its industry partners. It has organisational firewalls to protect it against any undue influences in its work or the publication of its findings. All industry partnerships are subject to review by an independent Scientific Advisory Committee or another independent review body, based on due diligence, TTPs and public sector requirements. FIND catalyses product development, leads evaluations, takes positions, and accelerates access to tools identified as serving its mission. It provides indirect support to industry (e.g. access to open specimen banks, a clinical trial platform, technical support, expertise, laboratory capacity strengthening in LMICs) to facilitate the development and use of products in these areas. FIND also supports the evaluation of prioritised assays and the early stages of implementation of WHO-approved (guidance and PQ) assays using donor grants. In order to carry out test validations and evaluations, has product evaluation agreements with several private sector companies for the diseases FIND works in which strictly define its independence and neutrality vis a vis the companies whose products get evaluated, and describes roles and responsibilities. R. Burton has nothing to disclose. A. Ward has nothing to disclose. M.P. Nicol reports grants from FIND, during the conduct of the study. G. Meintjes has nothing to disclose. C.M. Denkinger is a former employee of FIND. T. Broger reports grants from GHIT, personal fees from FIND, during the conduct of the study; and has a patent pending in the field of lipoarabinomannan detection for TB diagnosis.

Support statement: This work was funded by Global Health Innovative Technology (GHIT) Fund grant number G2015-201, UK Department for International Development (DFID) grant number 300341-102, Dutch Ministry of Foreign Affairs grant number PDP15CH14, Bill and Melinda Gates Foundation grant number OPP1105925, Australian Department of Foreign Affairs and Trade (DFAT) grant number 70957 and the German Federal Ministry of Education and Research (BMBF) through KfW. The Cohortl study was funded by the Wellcome Trust (088590 and 085251). B. Sossen received salary support from the Wellcome Trust (grant number 088316). G. Meintjes was supported by Wellcome Trust (098316 and 203135/Z/16/Z), the South African Research Chairs Initiative of the Department of Science and Technology and National Research Foundation (NRF) of South Africa (grant number 64787), NRF incentive funding (UID: 85858) and the South African Medical Research Council through its TB and HIV Collaborating Centres Programme, with funds received from the National Department of Health (RFA\#SAMRC-RFA-CC:TB/HIV/ AIDS-01-2014). C. Schultz received funding from the South African Medical Research Council through the National Health Scholarship Programme. A.D. Kerkhoff was supported by the National Institute of Allergy and Infectious Diseases (grant number T32 AI060530). The funders had no role in the study design, data collection, data analysis, data interpretation, or writing of this report. The opinions, findings and conclusions expressed in this manuscript reflect those of the authors alone.

\section{References}

1 Gupta RK, Lucas SB, Fielding KL, et al. Prevalence of tuberculosis in post-mortem studies of HIV-infected adults and children in resource-limited settings: a systematic review and meta-analysis. AIDS 2015; 29: 1987-2002. 
2 Kerkhoff AD, Barr DA, Burton R, et al. Disseminated tuberculosis among hospitalised HIV patients in South Africa: a common condition that can be rapidly diagnosed using urine-based assays. Sci Rep 2017; 7: 10931.

3 Cummings MJ, O'Donnell MR. Inverting the pyramid: increasing awareness of mycobacterial sepsis in sub-Saharan Africa. Int J Tuberc Lung Dis 2015; 19: 1128-1134.

4 World Health Organization. Xpert MTB/RIF assay for the diagnosis of pulmonary and extrapulmonary TB in adults and children. Geneva, World Health Organization, 2014.

5 Huerga H, Ferlazzo G, Bevilacqua P, et al. Incremental yield of including determine-TB LAM assay in diagnostic algorithms for hospitalized and ambulatory HIV-positive patients in Kenya. PLoS One 2017; 12: e0170976-15.

6 Boyles TH, Griesel R, Stewart A, et al. Incremental yield and cost of urine Determine TB-LAM and sputum induction in seriously ill adults with HIV. Int J Infect Dis 2018; 75: 67-73.

7 World Health Organization. High-priority target product profiles for new tuberculosis diagnostics: report of a consensus meeting. Geneva, World Health Organization, 2014.

8 Lawn SD. Point-of-care detection of lipoarabinomannan (LAM) in urine for diagnosis of HIV-associated tuberculosis: a state of the art review. BMC Infect Dis BioMed Central 2012; 12: 103.

9 Peter JG, Zijenah LS, Chanda D, et al. Effect on mortality of point-of-care, urine-based lipoarabinomannan testing to guide tuberculosis treatment initiation in HIV-positive hospital inpatients: a pragmatic, parallel-group, multicountry, open-label, randomised controlled trial. Lancet 2016; 387: 1187-1197.

10 Gupta-Wright A, Corbett EL, van Oosterhout JJ, et al. Rapid urine-based screening for tuberculosis in HIV-positive patients admitted to hospital in Africa (STAMP): a pragmatic, multicentre, parallel-group, double-blind, randomised controlled trial. Lancet 2018; 392: 292-301.

11 TAG. The LAM Test: vital for diagnosis TB in people living with advanced HIV. New York; 2017 p. 1-7.

12 Broger T, Sossen B, Toit du E, et al. Novel lipoarabinomannan point-of-care tuberculosis test for people with HIV: a diagnostic accuracy study. Lancet Infect Dis 2019; 19: 852-861.

13 Lawn SD, Kerkhoff AD, Burton R, et al. Rapid microbiological screening for tuberculosis in HIV-positive patients on the first day of acute hospital admission by systematic testing of urine samples using Xpert MTB/RIF: a prospective cohort in South Africa. BMC Med BioMed Central 2015; 13: 192.

14 Schutz C, Barr DA, Andrade BB, et al. Clinical, microbiologic, and immunologic determinants of mortality in hospitalized patients with HIV-associated tuberculosis: a prospective cohort study. PLoS Med 2019; 16: e1002840.

15 Broger T, Tsionksy M, Mathew A, et al. Sensitive electrochemiluminescence (ECL) immunoassays for detecting lipoarabinomannan (LAM) and ESAT-6 in urine and serum from tuberculosis patients. Wilkinson KA, editor. PLoS One 2019; 14: e0215443-19.

16 Lawn SD, Gupta-Wright A. Detection of lipoarabinomannan (LAM) in urine is indicative of disseminated TB with renal involvement in patients living with HIV and advanced immunodeficiency. Trans $R$ Soc Trop Med Hyg 2016; 110: 180-185. 